\title{
Identification of Serum Biomarkers in End Stage Liver Disease
}

\author{
D. Koutsogiannis ${ }^{1}$, K. Summers ${ }^{2}$, B. George ${ }^{1}$, P. Adams ${ }^{3}$, P. Marotta ${ }^{3}$ and S. Chakrabarti*, 1 \\ ${ }^{I}$ Departments of Pathology; ${ }^{2}$ microbiology and Immunology and ${ }^{3}$ Medicine, London Health Sciences Centre \& Univer- \\ sity of Western Ontario, London, Ontario, Canada
}

\begin{abstract}
Background: Progressive fibrosis and cirrhosis, clinically presenting as end-stage liver disease are common outcomes in alcoholic hepatitis as well as non-alcoholic fatty liver disease(NAFLD). In these processes, a series of changes occurs in liver tissues leading to cell death, remodeling, fibrosis and regeneration. The aim of this study is to identify potential novel biomarkers for non-invasive diagnosis of cirrhosis due to alcoholic etiology or NAFLD.

Methods: Serum from patients with biopsy proven end-stage liver disease of various etiologies, namely NAFLD(n=9), al$\operatorname{cohol}(n=5)$, and other end-stage liver diseases(n=6), who underwent liver transplant during the first six months of 2007 were utilized for retrospective analysis. Serum samples were also collected from a group of healthy volunteers $(n=7)$. The samples were analysed using Luminex technology or ELISA for 27 biomarkers that are known to be involved in pathologic processes such as cell death, regeneration and fibrosis.

Results: Of the 27 serum markers examined, 16 were elevated in the serum in all groups with end-stage liver diseases compared with the control group. They include adipokines, apoptosis and inflammatory mediators and growth factors. Interestingly, the serum of NAFLD patients showed significantly elevated HGF levels and trend towards increase in sFAS, TGF $\beta 1$, TNFR-1, TNFR-2 and leptin. The level of serum markers showed excellent correlation with each other indicating a complex interdependent pathogenetic mechanism.

Conclusions: The data from this study indicate that a large number of serum markers are altered in end-stage liver diseases. A panel of such markers may potentially be useful in assessing advanced fibrosis and cirrhosis in patients with chronic end stage liver diseases.
\end{abstract}

\section{INTRODUCTION}

Progressive fibrosis and ultimately cirrhosis are common features in a number of chronic end-stage liver diseases. In these processes, a series of changes occur in liver tissues leading to cell death, remodeling, regeneration and fibrosis. During this process of cellular death and regeneration, a series of alterations take place at the molecular and cellular level, leading to the alteration of a vast number of molecules. Hepatic stellate cells (HSCs) have been revealed as key components in hepatic healing and fibrosis [1]. There is emerging evidence that the fibrogenic function of HSCs may be influenced by cytokines, growth factors, death receptor ligands, and even adipokines [2-4].

From the diagnostic and prognostic aspect, percutaneous liver biopsy is considered the gold standard for the assessment of fibrosis. However, this procedure is expensive, invasive, amenable to sampling and interpretive error, and poses a considerable risk of complications including pneumothorax, hemorrhage, and puncture of other viscera. As a consequence to these limitations, a great deal of attention has been given to institute safe and reliable surrogate markers of liver fibrosis. In some of the earlier studies, alterations of serum levels of the molecules such as pro-collagen peptides, FAS,

*Address correspondence to this author at the Department of Pathology, The University of Western Ontario, London Health Sc. Ctr, 339 Winderemere Rd., London, Ontario, N6A 5A5, Canada; Tel: (519) 663-3381; Fax: (519) 663-2930; E-mail: subrata.chakrabarti@schulich.uwo.ca
TRAIL, and metalloproteinases have been demonstrated to correlate with degree of liver fibrosis [5-8].

Furthermore, in an end stage cirrhotic liver, histologic findings in conditions such as alcoholic liver diseases and non-alcoholic fatty liver disease (NAFLD) are similar. Hence, etiologic differentiation based on histologic findings is extremely difficult. Along with alcoholic liver diseases, NAFLD is becoming more common worldwide, possibly due to lifestyle alteration, increased incidence of obesity and metabolic syndrome. In the past 10 years, the rate of obesity has doubled in adults and tripled in children [9]. Both alcohol and NAFLD may ultimately lead to progressive cirrhosis and fibrosis. It is important to distinguish NAFLD from alcoholic liver disease as management between these two etiologies vastly differs.

Within the last decade, studies have attempted to evaluate non-invasive diagnostic tests for advanced liver disease such as fibrosis and cirrhosis using echography, transient elastography, magnetic resonance imaging, and biochemical tests [7-10]. Serum markers of fibrosis have the potential of either replacing the need for liver biopsy or identifying those patients with more advanced liver disease who may benefit from a liver biopsy and other subsequent intervention. Ideally, such markers should possess the ability to discriminate between degrees and etiologies of fibrosis and may even have the ability to determine response to various therapies and evaluate not only disease progression but also possible regression. 
The aim of this study was to identify potential novel serum biomarkers which may be useful in the diagnosis of advanced fibrosis and cirrhosis and specifically, to evaluate whether they are helpful in differentiating the etiologic factors such as alcohol or NAFLD.

\section{METHODS}

\section{Patient Population}

To determine the levels of the potential markers of liver fibrosis in patients with various liver diseases, we carried out a retrospective analysis. Pre transplantation serum from patients with biopsy proven end-stage liver diseases $(n=20)$ of various etiologies, who underwent consecutive liver transplants, during the first six months of 2007 were collected and stored at $-80^{\circ} \mathrm{C}$. These samples were utilized for analysis. They were grouped as alcoholic cirrhosis $(n=5)$, cirrhosis associated with NAFLD $(n=9)$. The other group consists of end stage liver disease of various etiologies not related to alcohol or $\operatorname{NAFLD}(n=6)$. Histopathological findings of the explanted liver were reviewed in a masked fashion by two pathologists, to confirm the diagnosis. All patients' charts were reviewed for possible clinical correlations with other relevant data such obesity, diabetes, and liver enzyme levels. No additional inclusion/exclusion criterias were used as this study was retrospective and exploratory in nature. Serum from healthy volunteers $(n=7)$, similarly collected and stored, were also analyzed. The volunteer (control) group consisted of 4 male and 3 females with age range of 32-55 years. They didn't have any known liver diseases and no history of alcoholism. They didn't show any sign of acute illness during the time of blood collection. Liver enzyme assessment or biopsies were not performed for this group.

\section{Quantification of Biomarkers}

We used Luminex ${ }^{\mathrm{TM}}$ (Luminex Corp., TX), fluorescence bead-based system to carry out simultaneous analysis of multiple biomarkers. Luminex uses a bead-based technology. Each bead set is coated with a antibody specific to a particular molecule. Within the analyzer, laser excites the internal dyes that identify each microsphere particle, and also captures any reporter dye during the assay. In this way, $\mathrm{xMAP}^{\mathrm{TM}}$ technology allows multiplexing of up to 100 unique quantitative assays within a single sample.

Serum was analyzed for levels of (1) inflammatory cytokines: interleukin (IL)-1ra, IL-4, IL-6, IL-8, IL-10, TNFR-1, TNFR-2, TNF- $\alpha$, IFN- $\alpha$ and C-reactive protein (CRP); (2) proteinases: MMP-1, MMP-2, MMP-9; (3) adipokines: adiponectin, leptin, resistin; (4) angiogenic and growth factors: HGF, bFGF, PDGF-bb, TGF-b, VEGF; (5) apoptosis markers: soluble Fas, Fas ligand (sFasL) using multiplexed biomarker immunoassay kits according to manufacturers' instruction (Biosource, Medicorp, Que; Lincoplex, Millipore

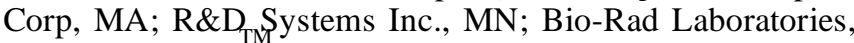
CA). A Bio-Plex 200 readout System was used (Bio-Rad). Levels were automatically calculated from standard curves using Bio-Plex Manager software (v.4.1.1, Bio-Rad). Levels of TGF- $\beta$ were measured using an enzyme-linked immunosorbent assay (ELISA) kit (BD Biosciences, ON).

\section{Statistical Analysis}

Statistical analyses were performed using Wilcoxon rank-sum test with post-hoc (bon-ferroni correction) analysis and Spearman correlation analysis.

\section{RESULTS}

The aim of this study was to identify potential serum markers that are altered in end-stage liver diseases. In addition we tried to identify whether the levels of any such markers are different in cirrhosis of various etiologies namely alcohol and NAFLD.

The demographic and the clinical data of the patient population are outlined in Table 1 . The age of the healthy

Table 1. Clinical Characteristics of the Patients in this Study

\begin{tabular}{|c|c|c|c|c|c|c|c|}
\hline Etiology & Age & Sex & Risk Factors & ALT & AST & Alk.phos & Bilirubin \\
\hline $\begin{array}{l}\text { Alcohol } \\
\text { Alcohol } \\
\text { Alcohol } \\
\text { Alcohol } \\
\text { Alcohol }\end{array}$ & $\begin{array}{l}66 \\
50 \\
58 \\
65 \\
41\end{array}$ & $\begin{array}{c}\text { Male } \\
\text { Male } \\
\text { Male } \\
\text { Male } \\
\text { Female }\end{array}$ & $\begin{array}{c}\text { None } \\
\text { Obesity (BMI:34.8) } \\
\text { None } \\
\text { None } \\
\text { DM, obesity (BMI: 31) }\end{array}$ & $\begin{array}{c}17 \\
717 \\
36 \\
30 \\
13\end{array}$ & $\begin{array}{c}43 \\
2114 \\
62 \\
70 \\
24\end{array}$ & $\begin{array}{c}52 \\
90 \\
143 \\
127 \\
86\end{array}$ & $\begin{array}{c}60 \\
71 \\
117 \\
272 \\
57\end{array}$ \\
\hline $\begin{array}{l}\text { NAFLD } \\
\text { NAFLD } \\
\text { NAFLD } \\
\text { NAFLD } \\
\text { NAFLD } \\
\text { NAFLD } \\
\text { NAFLD } \\
\text { NAFLD } \\
\text { NAFLD }\end{array}$ & $\begin{array}{l}59 \\
58 \\
40 \\
65 \\
62 \\
67 \\
46 \\
61 \\
47\end{array}$ & $\begin{array}{c}\text { Male } \\
\text { Male } \\
\text { Male } \\
\text { Male } \\
\text { Female } \\
\text { Male } \\
\text { Male } \\
\text { Female } \\
\text { Female }\end{array}$ & $\begin{array}{c}\text { DM, obesity (BMI: 31) } \\
\text { DM } \\
\text { DM, obesity } \\
\text { Obesity (BMI: } 38.4 \text { ) } \\
\text { DM, obesity } \\
\text { DM, obesity (BMI: 28.8) } \\
\text { Obesity (BMI: 44.8) } \\
\text { Hypothyroidism, obesity } \\
\text { obesity (BMI: 36.6) }\end{array}$ & $\begin{array}{c}36 \\
404 \\
348 \\
21 \\
33 \\
33 \\
44 \\
33 \\
24\end{array}$ & $\begin{array}{c}78 \\
652 \\
487 \\
56 \\
48 \\
64 \\
80 \\
53 \\
37\end{array}$ & $\begin{array}{c}119 \\
202 \\
104 \\
74 \\
96 \\
93 \\
151 \\
119 \\
122\end{array}$ & $\begin{array}{c}154 \\
165 \\
166 \\
643 \\
27 \\
171 \\
71 \\
33 \\
37\end{array}$ \\
\hline $\begin{array}{l}\text { Budd-Chiari } \\
\text { Acetaminophen } \\
\text { Polycystic liver } \\
\text { Sarcoidosis } \\
\text { A-1 antitrypsin } \\
\text { Cryptogenic }\end{array}$ & $\begin{array}{l}35 \\
17 \\
53 \\
56 \\
65 \\
50\end{array}$ & $\begin{array}{c}\text { Male } \\
\text { Female } \\
\text { Female } \\
\text { Female } \\
\text { Male } \\
\text { Male }\end{array}$ & $\begin{array}{c}\text { Methadone abuse } \\
\text { None } \\
\text { None } \\
\text { Obesity (BMI:31.7) } \\
\text { DM } \\
\text { None }\end{array}$ & $\begin{array}{c}26 \\
4006 \\
20 \\
15 \\
31 \\
44\end{array}$ & $\begin{array}{c}56 \\
2032 \\
34 \\
24 \\
64 \\
80\end{array}$ & $\begin{array}{l}163 \\
211 \\
138 \\
138 \\
174 \\
151\end{array}$ & $\begin{array}{l}34 \\
86 \\
12 \\
52 \\
71 \\
72\end{array}$ \\
\hline
\end{tabular}


Table 2.

\begin{tabular}{|c|c|c|c|c|c|c|c|c|c|c|c|c|c|}
\hline \multicolumn{14}{|l|}{ GROUPS } \\
\hline $\begin{array}{l}\text { BIO- } \\
\text { MARKERS }\end{array}$ & Units & \multicolumn{4}{|l|}{ NAFLD } & \multicolumn{3}{|c|}{ ALCOHOL } & \multicolumn{3}{|l|}{ OTHERS } & \multicolumn{2}{|c|}{ CONTROL } \\
\hline \multicolumn{14}{|c|}{ Apoptosis related } \\
\hline sFas & $\mathrm{pg} / \mathrm{ml}$ & 15072.7 & 14756.3 & $4194.4 \$$ & 10320.3 & 7786.6 & 3085.4 & 11213.1 & 10796.1 & 4034.1 & 4559.7 & 4676.2 & $473.8^{*}$ \\
\hline \multicolumn{14}{|c|}{ Growth Factors } \\
\hline TGF- $\beta 1$ & $\mathrm{ng} / \mathrm{ml}$ & 3.9 & 2.6 & $3.4 \$$ & 1.5 & 1.5 & 1.9 & 2.6 & 0.9 & 3.4 & 0 & 0 & $0^{*}$ \\
\hline TGF- $\alpha$ & $\mathrm{pg} / \mathrm{ml}$ & 149.3 & 42.6 & 296.8 & 1187.4 & 30.3 & 2804.9 & 156.7 & 45.64 & 280.5 & 68.7 & 57.8 & 56.6 \\
\hline HGF & $\mathrm{pg} / \mathrm{ml}$ & 753.6 & 767.9 & $165.4 * *$ & 523.7 & 478 & 127.8 & 680.3 & 503.4 & 453.7 & 131.8 & 124.5 & $28.5^{*}$ \\
\hline \multicolumn{14}{|c|}{ Inflammatory mediators } \\
\hline IFN- $\alpha$ & $\mathrm{pg} / \mathrm{ml}$ & 27.7 & 7.7 & 19.3 & 32.6 & 0.5 & 55.2 & 25.6 & 0 & 52.5 & 15.2 & 0 & 26 \\
\hline TNFR-1 & $\mathrm{pg} / \mathrm{ml}$ & 7175.3 & 4209.9 & $6586.2 \$$ & 2632.5 & 2552.2 & 1164.9 & 2612.9 & 2388.3 & 1124 & 621.4 & 614.5 & $161.5^{*}$ \\
\hline TNFR-2 & $\mathrm{pg} / \mathrm{ml}$ & 6784.7 & 6824.8 & $4025.3 \$$ & 3472.8 & 2816.5 & 1756.6 & 4011.4 & 3833.1 & 1200.2 & 1265.1 & 1248.1 & $339.1 *$ \\
\hline IL-1 $\beta$ & $\mathrm{pg} / \mathrm{ml}$ & 16 & 0 & 25.3 & 6 & 0 & 14.7 & 0 & 0 & 0 & 0 & 0 & 0 \\
\hline IL-1ra & $\mathrm{pg} / \mathrm{ml}$ & 360.4 & 359.1 & 171.5 & 235.2 & 186.7 & 208 & 135.1 & 74.4 & 168.3 & 0 & 0 & \\
\hline $\mathrm{IL}-1 \alpha$ & $\mathrm{pg} / \mathrm{ml}$ & 1041.6 & 31.6 & 2322.9 & 1054.1 & 424.7 & 1857.9 & 987.5 & 811.9 & 342.6 & 2569.6 & 2405.4 & 2703.8 \\
\hline IL-4 & $\mathrm{pg} / \mathrm{ml}$ & 2 & 2 & 1.1 & 2 & 1.3 & 0.9 & 2.4 & 2.2 & 1.6 & 0.2 & 0 & $0.5^{*}$ \\
\hline CRP & $\mathrm{ng} / \mathrm{ml}$ & 20221.2 & 23000 & 9075.7 & 15712.8 & 15712.8 & 11289.3 & 23000 & 23000 & 0 & 7090.5 & 1300.1 & 10877.5* \\
\hline MMP-1 & $\mathrm{pg} / \mathrm{ml}$ & 3959.8 & 3335.9 & 2555.9 & 4617 & 2955.8 & 3618.8 & 4628.9 & 4187.8 & 2903.8 & 1484.6 & 1402.2 & $679.4 *$ \\
\hline MMP-2 & $\mathrm{ng} / \mathrm{ml}$ & 336.1 & 297 & 85.4 & 329.9 & 329.9 & 71.7 & 264.3 & 250 & 73.8 & 177.2 & 173.7 & $20.6^{*}$ \\
\hline MMP-9 & $\mathrm{ng} / \mathrm{ml}$ & 321.1 & 285.3 & 86.8 & 357.1 & 326.4 & 147.3 & 355.3 & 273.9 & 248.8 & 44 & 41.7 & $17 *$ \\
\hline \multicolumn{14}{|c|}{ Adipocytokines } \\
\hline Leptin & $\mathrm{pg} / \mathrm{ml}$ & 18986.3 & 7976.1 & 6318.9 & 25055.7 & 24300.2 & 16426.8 & 23994 & 20002.2 & 12792.7 & 15508 & 7406.2 & 17651.5 \\
\hline Resistin & $\mathrm{pg} / \mathrm{ml}$ & 17802.8 & 17468.5 & 4908.3 & 25640.4 & 17599.5 & 17599.5 & 12025.2 & 10376.5 & 4259.3 & 6548.1 & 6731.5 & $1801.8^{*}$ \\
\hline Adiponectin & $\mathrm{ng} / \mathrm{ml}$ & 15856.3 & 17245.3 & 7337.8 & 19153.8 & 18706 & 6478 & 19567.9 & 21092.33 & 5109.7 & 8484 & 8562.7 & $1837.2^{*}$ \\
\hline
\end{tabular}

$*$ = significantly different compared to all other groups or Alcohol and NASH group combined, $* *=$ significantly different compared to alcohol group, $\$=$ p $<0.2$ compared to alcohol group.

volunteers ranged from 29-51 $(\mathrm{M}=4, \mathrm{~F}=3)$. None of the volunteers had any history of diabetes, liver disease or alcoholism.

Of the 27 biomarkers examined, 16 were significantly elevated (2.7-to $>2000$ fold in mean value) in the serum of the patients with all end-stage liver diseases (irrespective of etiology) were compared with the control group (Table 2). Similar statistical significance was also observed when control group were compared with cirrhotic patients of com- bined alcohol and NAFLD group. These biomarkers include adipocytokines, markers of apoptosis, inflammation and growth factors. Both sFas and sFas ligands were significantly elevated. Among the growth factors, TGF $\beta 1$ and HGF were significantly elevated. No alteration in TGF $\alpha$ and bFGF were seen. Although both VEGF (4.3 fold) and PDGFbb (1.9 fold) were elevated, the $\mathrm{p}$ values fell short of significance ( $p<0.06$ and $<0.07$ respectively). Several of the inflammatory cytokines were significantly elevated in the cirrhotic livers. These include TNFR1, TNFR2, IL1ra, IL-4, -6, -8, MMP-1, 
$-2,-9$ an CRP. No significant alterations were seen in IL- $1 \alpha$, IL-10, -13. In the adipocytokine group both adiponectin and resistin were significantly elevated in all groups with endstage liver diseases, whereas no significant alterations in serum leptin levels were observed. Very high levels of upregulation were seen in sFas ligand, IL-1ra and IL-6 in these groups compared to controls. It is of further interest to note that some of the investigated molecules such as TGF $\beta 1$, IL-6, IL-1B and IL-1ra were undetectable in the control population.

A further intention of the study was to observe if any patterns of markers were able to differentiate two specific common etiologies of cirrhosis. In this respect, the serum of NAFLD patients showed significantly $(\mathrm{p}<0.01)$ elevated HGF levels. It is however to be noted that several biomarkers, such as sfas, TGF $\beta 1$, TNFR-1, -2 and leptin, were different in these two groups. However these failed to reach a level of statistical significance and they demonstrated a $\mathrm{p}$ value ranging from $<0.1$ to $<0.2$.
The correlation analysis showed excellent correlation of various biomarkers with each other. Except for leptin and IL10 (undetectable in any group), all other biomarkers showed significant correlation with one or more markers from same and other groups (Table $\mathbf{3}$ ).

\section{DISCUSSION}

Liver biopsy utilized to stage liver diseases has recently become increasingly being criticized because of its invasiveness and expense, despite the associated low incidence of morbidity and mortality [10]. The major drawback of liver biopsy is, however, the potential for sampling error. A liver biopsy samples a miniscule portion of liver; and even with a $2.5 \mathrm{~cm}$ biopsy, inaccurate staging can occur in up to $20 \%$ of biopsy samples [11].

Currently, there are multiple non-invasive methodologies proposed to evaluate liver fibrosis and all appear to perform reasonably well and with a similar diagnostic accuracy. The FibroTest (FibroSure, LabCorp.USA) and FIBROSpect II

\section{Table 3. Spearman Rank Correlation}

\begin{tabular}{|c|c|}
\hline Biomarkers & Correlates significantly $(\mathbf{p}<0.05)$ with \\
\hline \multicolumn{2}{|c|}{ Apoptosis related } \\
\hline sFas & sFas ligand, TGF- $\beta 1$, HGF, VEGF, TNFR-1, TNFR-2, IL-1B, IL1ra, IL-4, IL-6, IL-8, MMP-2, resistin, adiponectin \\
\hline sFas ligand & S Fas, TGF- $\beta$ 1, HGF, TNFR-1, TNFR-2, IL-8, MMP-2, \\
\hline \multicolumn{2}{|c|}{ Growth Factors } \\
\hline TGF- $\beta 1$ & S Fas, sFas ligand, HGF, IFN $\alpha$, TNFR-1, TNFR-2, IL1ra, IL-4, IL-6,MMP-2 \\
\hline TGF- $\alpha$ & basic FGF, IL-1 $\alpha$, adiponectin \\
\hline HGF & S fas, sFas ligand, TGF- $\beta 1$, VEGF, TNFR-1, TNFR-2, IL-6, IL-8, MMP-2, MMP-9, resistin, CRP, adiponectin \\
\hline Basic FGF & Il-10, IL-13, TNF $\alpha$ \\
\hline PDGF-bb & VEGF, IL1ra, MMP-1, MMP-9 \\
\hline VEGF & sFas, HGF, PDGF-bb, IFN $\alpha$, TNFR-1, TNFR-2, IL-6, IL-8, MMP-9, resistin, CRP \\
\hline \multicolumn{2}{|c|}{ Inflammatory mediators } \\
\hline IFN- $\alpha$ & TGF- $\beta 1$, PDGF-bb, VEGF, \\
\hline TNFR-1 & sFas, sFas ligand, TGF- $\beta 1$, HGF, VEGF, TNFR-2, IL1ra, IL-4, IL-6,MMP-2, MMP-9, leptin, resistin \\
\hline TNFR-2 & sFAS, sFas ligand, TGF- $\beta 1$, HGF, VEGF, TNFR-1, IL1ra, IL-6, MMP-9, leptin, resistin \\
\hline IL-1 $\beta$ & IL-6, IL-8 \\
\hline IL-1 ra & sFas, TGF- $\beta 1$, HGF, TNFR-1, TNFR-2, IL-4, IL-6 \\
\hline IL-1 $\alpha$ & TGF- $\alpha$ \\
\hline IL-4 & sFas, TGF- $\beta 1$, PDGF-bb, TNFR-1, IL1ra, IL-6,IL-13, MMP-1, MMP-2, MMP-9, resistin, adiponectin \\
\hline IL-6 & sFas, TGF- $\beta 1$, HGF, VEGF, TNFR-1, TNFR-2, IL-1 $\beta, I L 1$ ra, IL-4, IL-8, MMP-2, MMP-9, resistin, CRP, adiponectin \\
\hline IL-8 & S Fas, sFas ligand, HGF,VEGF, TNFR-1, TNFR-2, IL-1 $\beta$,IL1ra, IL-4, MMP-1, MMP-2, MMP-9, resistin, CRP, adiponectin \\
\hline IL-10 & - \\
\hline IL-13 & basic FGF, IL-4, TNF- $\alpha$ \\
\hline TNF- $\alpha$ & basic FGF, IL-1ra MMP-1 \\
\hline MMP-1 & PDGF-bb,IL-4,IL-8, TNF- $\alpha$ \\
\hline MMP-2 & S Fas, sFas ligand, TGF- $\beta$ 1,HGF, TNFR-1,IL-1ra,IL-4,IL-6,IL-8, IL-9, resistin, CRP, adiponectin \\
\hline MMP-9 & HGF, PDGF-bb, VEGF, TNFR-1,TNFR-2,IL-1ra,IL-4, IL-6,IL-8, MMP-2, MMP-9, resistin, CRP, adiponectin \\
\hline CRP & HGF, VEGF, TNFR-1,TNFR-2, IL-6,IL-8, MMP-2, MMP-9, resistin, adiponectin \\
\hline \multicolumn{2}{|c|}{ Adipocytokines } \\
\hline Leptin & - \\
\hline Resistin & sFas, HGF, VEGF, TNFR-1,TNFR-2, IL-1ra,IL-4, IL-6,IL-8, MMP-2, MMP-9,CRP \\
\hline Adiponectin & sFas, HGF, TG F- $\alpha$, IL-4, IL-6,IL-8, MMP-1,MMP-2, MMP-9,CRP \\
\hline
\end{tabular}


(Prometheus Laboratories Inc., San Diego, CA) are commonly utilized tests that combine multiple markers for predictive value, including hyaluronic acid, tissue inhibitor of metalloproteinase-1 (TIMP-1), haptoglobin, $\alpha_{2}$ macroglobulin, apolipoprotein A1, and total bilirubin [12]. A recent study from Rosenberg and the European Liver Fibrosis Group also used HA, procollagen peptide III and TIMP-1 to stage fibrosis in a variety of liver diseases with promising results [13]. Moreover, the SHASTA index (using Serum HA, AST, Albumin) was also shown to predict fibrosis [14].

The data from this study indicate that the large number of serum markers, involved in various processes such as apoptosis, fibrosis and angiogenesis, are altered in cirrhosis; and a panel of such markers may potentially be useful in assessing end-stage liver damage and cirrhosis in patients with chronic liver diseases. Use of luminex technology provides an unique opportunity to quantify multiple molecules from a small sample and provides a novel avenue to explore a large number of molecules. This technology also allowed us to measure previously undetectable levels of proteins. Such technology has previously been used in other disease process [15]. Although several of the molecules we studied may mediate several cellular processes, based on some of their major functions we selected to study specific markers as they relate to specific pathogenetic processes of interest in cirrhosis. We found alterations of several biomarkers. The results of this study are in keeping with the complexity of the pathogenetic mechanisms, such as cell death, inflammation, angiogenesis and fibrosis leading to cirrhosis. The correlation analysis further suggests interactions of these factors and complexity of the process regardless of its etiology. Some of our data are in keeping with the previous findings whereas others are not. Previous studies have shown increased TGF $\beta 1$, IL-10, TNFR-2, IL-8, MMP-1, MMP-2, MMP-9 in cirrhosis [7, 1619]. However, majority of such studies were carried out in hepatitis $C$ patients. In our study we found increased TGF $\beta 1$, TNFR-2, IL8, MMP-1,-2,-9 in all cirrhotic patients regardless of etiology. We didn't detect increased IL-10 in any groups in this patient population of non-viral end-stage liver disease. Some investigators have also demonstrated increased IL-8 and IL-6 in alcoholic liver disease and suggested that this can be used as a marker for such etiology $[20,21]$. None of these studies however, compared the levels of these markers with that in the cirrhosis of non-alcoholic etiology. In our study, these biomarkers were elevated in end stage liver disease irrespective of the etiology. Hence although, they may be useful to assess extent of the liver damage (as in normal volunteer, they were very low or undetectable), they may not be useful as etiology-specific marker. Similarly increased serum adiponectin and resistin levels have previously demonstrated in NAFLD and have been proposed to be a potential markers of these diseases [22, 23]. However, these studies also didn't compare these data with liver damage induced by other etiologic factors. In our study such increase were seen in all end stage liver diseases. Hence these markers may not so be etiology specific.

Based on this study measurement of a panel of markers including $\mathrm{sFas}$ and $\mathrm{sFas}$ ligands, TGF $\beta 1$, HGF, TNFR-1, TNFR-2, IL-1ra, IL4, IL-6, IL-8, MMP-1, MMP-2, MMP-9, CRP, adiponectin and resistin may be useful in the diagnosis of end stage liver disease such as cirrhosis. However, a smaller panel including the molecules such as sFAS ligand,
TGF $\beta 1$, IL-1ra and IL-6 which are either undetectable in normal population or highly elevated in cirrhosis may be a practical alternative. However, larger studies are necessary to confirm such notion. It is also interesting to note that levels of HGF were significantly different in Alcohol and NAAFLD group. Increased HGF has previously been demonstrated in alcoholic hepatitis [24]. Similar to our study, in chronic liver disease, it has been shown to correlate with CRP [25]. In addition, we have also observed that several other biomarkers such as sFas, TGF $\beta 1$, TNFR-1, -2 and leptin showed a trend to increase in alcoholic cirrhosis compared to NAFLD group. . Although, these didn't reach the level of statistical significance $(p<0.1-p<0.2)$, possibly due to a small sample size, further studies are warranted. Hence in is tempting to speculate that a panel including such marker may be useful for future studies to consider, which may potentially be helpful in differentiation between these two etiologic factors. We refrained from carrying out additional analysis to identify predictive values of the markers identified in this study as we recognized the limitation, i.e, small sample size. However, larger prospective studies in patients with chronic liver disease may be conducted to confirm the accuracy of these markers. Such studies are already underway in our laboratories. Nevertheless, the present study may represent a first step towards the identification of a panel of such non-invasive serum markers. As the knowledge of biomarkers and noninvasive tests for fibrosis increases and long-term studies become available, it will be interesting to study if these tests have the same prognostic value as liver biopsy in relation to the risk of disease progression and clinical outcomes.

\section{ACKNOWLEDGEMENTS}

The study was supported by funds from the division of gastroenterology, London Health Sciences Centre. We also acknowledge the valuable help from Mr. Larry Stitt of Biostatistical support unit, The University of Western Ontario.
ABBREVIATIONS

$\begin{array}{ll}\text { AST } & =\text { Aspartate transaminase } \\ \mathrm{ALT} & =\text { Alanine transaminase } \\ \mathrm{bFGF} & =\text { Basic fibroblast growth factor } \\ \mathrm{CRP} & =\mathrm{c} \text { - reactive protein } \\ \mathrm{DM} & =\text { Diabetes mellitus } \\ \text { ELISA } & =\text { Enzyme-linked immunoabsorbant assay } \\ \mathrm{IFN} & =\text { Interferon } \\ \mathrm{IL} & =\text { Interleukin } \\ \text { MMP } & =\text { Matrix metalloproteinase } \\ \text { NAFLD } & =\text { Non-alcoholic fatty liver disease } \\ \text { PDGF-bb } & =\text { Platelet derived growth factor }-\mathrm{bb} \\ \text { sFas } & =\text { Soluble Fas } \\ \text { TGF- } \beta & =\text { Transforming growth factor } \beta \\ \text { TNF } & =\text { Tumour necrosis factor } \\ \text { TNFR } & =\text { Tumour necrosis factor receptor } \\ \text { VEGF } & =\text { Vascular endothelial growth factor }\end{array}$




\section{REFERENCES}

[1] Ruddel RG, Oakley F, Hussain Z, et al. A role for serotonin in hepatic stellate cell function and liver fibrosis. Am J Pathol 2006; 169: 861-76.

[2] Elsharkawy AM, Oakley F, Mann DA. The role and regulation of hepatic stellate cell apoptosis in reversal of liver fibrosis. Apoptosis 2005; 10: 927-39.

[3] Lieber CS, Weiss DG, Paronetto F. Value of fibrosis markers for staging liver fibrosis in patients with precirrhotic alcoholic liver disease. Alchol Clin Exp Res 2008; 32: 1031-9.

[4] Saxena NK, Titus MA, Ding X, et al. Leptin as a novel profibrogenic cytokine in hepatic stellate cells. FASEB J 2004; 18: 1612-4.

[5] Lydatakis H, Hager IP, Kostadelou E, Mpousmpoulas S, Pappas S, Diamantis I. Non-invasive markers to predict the liver fibrosis in non-alcoholic fatty liver disease. Liver Int 2006; 26: 864-71.

[6] Luo R, Yang S, Xie J, Zhao Z, He Y, Yao J. Diagnostic value of five serum markers for liver fibrosis. Zhonghua Gan Zang Bing Za Zhi 2001; 9: 148-50.

[7] Sakugawa H, Nakayoshi T, Kobashigawa K, et al. Clinical usefulness of biochemical markers of liver fibrosis in patients with nonalcoholic fatty liver disease. World J Gastroenterol 2005; 11: 255-9.

[8] Poynard T, Ratziu V, Charlotte F, et al. Diagnostic value of biochemical markers (NashTest) for the prediction of non alcoholo steato hepatitis in patients with non-alcoholic fatty liver disease. BMC Gastroenterol 2006; 10: 6-34.

[9] National Institute of Diabetes and Digestive and Kidney Diseases, National Institutes of Health. Nonalcoholic Steatohepatitis. NIH 2006; Publication No. 07-4921,

[10] Afdhal NH. Diagnosing fibrosis in hepatitis C: is the pendulum swinging from biopsy to blood tests? Hepatology 2003; 37: 972-4.

[11] Bedossa P, Dargère D, Paradis V. Sampling variability of liver fibrosis in chronic hepatitis C. Hepatol 2003; 38: 1449-57.

[12] Mehta P, Ploutz-Snyder R, Nandi J, Rawlins S, Sanderson SO, Levine RA. Diagnostic accuracy of serum hyaluronic acid, FIBROSpectII, and YKL-40 for discriminating fibrosis stages in chronic hepatitis C. Am J Gastroenterol 2008; 103: 928-36.

[13] Rosenberg WM, Voelker M, Thiel R, et al. Serum markers detect the presence of liver fibrosis: a cohort study. Gastroenterology 2004; 127: 1704-13.

[14] Kelleher TB, Mehtaa SH, Bhaskar R, et al. Prediction of hepatic fibrosis in $\mathrm{HIV} / \mathrm{HCV}$ co-infected patients using serum fibrosis markers: the SHASTA index. J Hepatol 2005; 43: 78-84.
[15] Linkov F, Lisovich A, Yurkovetsky Z, et al. Early detection of head and neck cancer: development of a novel screening tool using multiplexed immunobead-based biomarker profiling. Cancer Epidemiol Biomarkers Prev 2007: 16: 102-7.

[16] Verma V, Chakravarti A, Kar P. Cytokine levels of TGF- $\beta$, IL-10, and sTNFalphaRII in type $\mathrm{C}$ chronic liver disease. Dig Dis Sci 2008; 53: 2233-7.

[17] Jarrar MH, Baranova A, Collantes R, et al. Adipokines and cytokines in non-alcoholic fatty liver disease. Aliment Pharmacol Ther 2008; 27: 412-21.

[18] Leroy V, Monier F, Bottari S, et al. Circulating matrix metalloproteinases 1, 2, 9 and their inhibitors TIMP-1 and TIMP-2 as serum markers of liver fibrosis in patients with chronic hepatitis C: comparison with PIIINP and hyaluronic acid. Am J Gastroenterol 2004; 99: 271-9.

[19] Grünhage F, Rezori B, Neef M, et al. Elevated soluble tumor necrosis factor receptor 75 concentrations identify patients with liver cirrhosis at risk of death. Clin Gastroenterol Hepatol 2008; 6: 125562 .

[20] Swiatkowska-Stodulska R, Bakowska A, Drobińska-Jurowiecka A. Interleukin-8 in the blood serum of patients with alcoholic liver disease. Med Sci Monit 2006; 12: CR215-20.

[21] Liappas IA, Nicolaou C, Chatzipanagiotou S, et al. Vitamin B12 and hepatic enzyme serum levels correlate with interleukin-6 in alcohol-dependent individuals without liver disease. Clin Biochem 2007; 40: 781-6.

[22] Zou CC, Liang L, Hong F, Fu JF, Zhao ZY. Serum adiponectin, resistin levels and non-alcoholic fatty liver disease in obese children. Endocr J 2005; 52: 519-24

[23] Pagano C, Soardo G, Pilon C, et al. Increased serum resistin in nonalcoholic fatty liver disease is related to liver disease severity and not to insulin resistance. J Clin Endocrinol Metab 2006; 91: 1081-6.

[24] Taïeb J, Delarche C, Paradis V, et al. Polymorphonuclear neutrophils are a source of hepatocyte growth factor in patients with severe alcoholic hepatitis. J Hepatol 2002; 36: 342-8.

[25] Shiota G, Umeki K, Okano J, Kawasaki H. Hepatocyte growth factor and acute phase proteins in patients with chronic liver diseases. J Med 1995; 26: 295-308.

(C) Koutsogiannis et al.; Licensee Bentham Open.

This is an open access article licensed under the terms of the Creative Commons Attribution Non-Commercial License (http://creativecommons.org/licenses/by-nc/3.0/) which permits unrestricted, non-commercial use, distribution and reproduction in any medium, provided the work is properly cited. 